\title{
Coupling Characteristics and Optimization of the Resonators Applied to the Power Supply System of Tail-free Household Appliances
}

\author{
Daohong Lin*, Wangcheng Zhu and Zhenchao Xie \\ Electric Power Research Institute of Hainan Power Grid Co., Ltd., Haikou 570125, Hainan Province, China \\ ${ }^{*}$ Corresponding author
}

\begin{abstract}
As household appliances become more and more intelligent, increasingly strict demands are put forward for their power supply. Existing draw-out power supply mode of household appliances has many shortcomings including low waterproof grade, touch spark, and wire constraint. Considering this, a tail-free power supply system based on magneticallycoupled resonant wireless power transfer (MCRWPT) technology is investigated. Aiming at the coupling problems, such as frequency splitting, of the resonator during the design of the system, the analytic calculation is adopted to obtain the advisable domain values of mutual inductance that can evade frequency splitting. On this basis, the authors also carry out theoretical calculation on the combination modes of disc spiral coils and spatial spiral coils so as to attain the accurate mutual inductance between the resonators. In addition, a calculation method more accurate than the traditional Neumann formula is also proposed. The theoretical analysis is finally validated by conducting simulation and experiments and comparing the obtained results.
\end{abstract}

Keywords-tail-free household appliances; wireless power transfer; resonator; frequency splitting; mutual inductance calculation

\section{INTRODUCTION}

With the constant innovation of the technologies in emerging and manufacturing industries, household appliances are developed to be intelligent and somatosensory so as to have high user experience. However, the traditional draw-out power supply mode limits the flexibility, reliability, and safety in power utilization of household appliances [1]. Moreover, frequently drawing out and inserting plugs is likely to cause problems relating safety utilization of electricity such as aging of contact elements, loose contact, and sparks. For this reason, it is urgent for experts in the field of household appliances to develop a new power supply mode, so as to cast off the last "tail" of the household appliances-wires [2].

Wireless power transfer (WPT) technology, as a nontraditional power transmission mode emerging in recent years, adopts electromagnetic coupling to realize isolated power supply of electric equipments. The WPT technology was firstly investigated by Croatia American physicist Nikola Tesla, while the presence of relevant bottleneck technical issues restricts the development of the technology [3]. In 2007, Marin Soljacic and his research team in Massachusetts Institute of Technology put forward the magnetically-coupled resonant wireless power transfer (MCRWPT) technology, which provides an opportunity for the development of high-power and mid-range WPT technology [4]. Since then, research teams in China and abroad have vigorously explored and solved key problems of MCRWPT, for example the increase in the transmission distance, the establishment of the accurate model of the system, and the further optimization of the efficiency and power. Meanwhile, great progress has been obtained [5-8]. The continuous development of the MCRWPT technology provides a new solution to the intelligent and green power supply for household appliances [9].

Through modeling analysis of the power supply system of tail-free household appliances, the coupling problems involving frequency splitting that need to be considered in the design of the system is studied using the KVL circuit analysis method converted by equivalent impedance. Moreover, the advisable domain values of the mutual inductance which can avoid the occurrence of frequency splitting of the power supply system are obtained. The value of mutual inductance is not only related to whether frequency splitting will occur or not, but also is associated with whether the system has optimal transmission performance or not. Therefore, it is very necessary to maintain the mutual inductance in the critical saturated state under conditions with a fixed transmission distance. However, the commonly-used analytic calculation methods at present are relatively rough, such that the theoretical mutual inductance cannot meet the requirement for maintaining in the critical saturated state. Therefore, an accurate calculation method is proposed for mutual inductance between resonance coils in the wireless power supply system for tail-free household appliances. Finally, the theoretical analysis of the research is validated through experimental measurement and comparison. The research result is of great practical significance for avoiding the occurrence of frequency splitting while improving the transmission performance of the system.

\section{THEORETICAL MODELING AND COUPLING CHARACTERISTICS OF THE SYSTEM}

From the perspective of power types, MCRWPT systems can be classified into voltage-source (V) and current-source (I) types. While according to the capacitance compensation approaches of transmit and receiving coils, the systems can be divided into four types: serial-serial (SS), serial-parallel (SP), parallel-serial (PS), and parallel-parallel (PP) ones. Therefore, 
through permutation and combination, there are eight types of resonant topology of MCRWPT systems [10-11]. Taking a MCRWPT system with V-source power and SS compensation topology for an example, the structure of the power supply system is shown in Figure I.

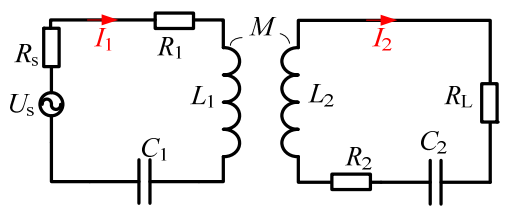

FIGURE I. WIRELESS POWER SUPPLY SYSTEM FOR HOUSEHOLD APPLIANCES

In Figure I, $U_{\mathrm{s}}$ represents the non-ideal voltage source, and its internal resistance is $R_{\mathrm{s}}$. Moreover, $L_{1}, R_{1}$, and $C_{1}$ denote self-inductance, internal resistance, and resonant compensation capacitance of the transmit coil; while $L_{2}, R_{2}$, and $C_{2}$ are those of the receiving coil, respectively. By using the KVL circuit analysis method converted by equivalent impedance, the equivalent input impedance in the power source is

$$
Z_{\text {in }}=R_{s}+R_{1}+j\left(\omega L_{1}-\frac{1}{\omega C_{1}}\right)+\frac{(\omega M)^{2}}{R_{L}+R_{2}+j\left(\omega L_{2}-\frac{1}{\omega C_{2}}\right)}
$$

When the system is in the resonant state, the imaginary part of the input impedance should be 0 , that is,

$$
\left(\omega L_{1}-\frac{1}{\omega C_{1}}\right)-\frac{(\omega M)^{2}\left(\omega L_{2}-\frac{1}{\omega C_{2}}\right)}{\left(R_{L}+R_{2}\right)^{2}+\left(\omega L_{2}-\frac{1}{\omega C_{2}}\right)}=0
$$

Suppose that the transmit and receiving coils have same parameters and natural resonant frequency, namely,

$$
\left\{\begin{array}{l}
L_{1}=L_{2}=L \\
C_{1}=C_{2}=C \\
1 / \sqrt{L_{1} C_{1}}=1 / \sqrt{L_{2} C_{2}}=\omega
\end{array}\right.
$$

Therefore, Formula (2) can be further simplified as

$$
\left(\omega L-\frac{1}{\omega C}\right)\left[\left(R_{L}+R_{2}\right)^{2}+\left(\omega L-\frac{1}{\omega C}\right)^{2}-(\omega M)^{2}\right]=0
$$

Two solutions to the resonant frequency can be obtained by solving Formula (4), i.e. making either the first or second part zero. There is a real solution in the solution set and the other one needs to undergo discrimination of the root according to the actual parameters. Therefore, the determined real solution, that is, $\omega=1 / \sqrt{L C}$ is the natural resonant frequency of the wireless power supply system of household appliances. When the second part in Formula (4) is zero, the formula can be solved based on the quadratic equation of one variable as

$$
\omega_{1,2}=\sqrt{\frac{-\left[\left(R_{L}+R_{2}\right)^{2}-\frac{2 L}{C}\right] \pm \sqrt{\Delta}}{2\left(L^{2}-M^{2}\right)}}
$$

where $\Delta=\left[\left(R_{L}+R_{2}\right)^{2}-\frac{2 L}{C}\right]^{2}-\frac{4\left(L^{2}-M^{2}\right)}{C^{2}} \quad$ is the discriminant of the root. In many cases, the self-inductance of coils is larger than the mutual inductance between coils. Therefore, if Formula (5) has a real solution, it needs to meet the following constraints:

$$
\left\{\begin{array}{l}
\Delta \geq 0 \\
\left(R_{L}+R_{2}\right)^{2}-\frac{2 L}{C} \leq 0
\end{array} .\right.
$$

The above analysis reveals that when the transmit and receiving coils of the MCRWPT system have a large distance, the mutual inductance is ignorable compared with the selfinductance, so $4\left(L^{2}-M^{2}\right) / C^{2} \approx 4 L^{2} / C^{2}$. Therefore, the discriminant can be further rewritten as

$$
\Delta \approx\left(R_{L}+R_{2}\right)^{2}\left[\left(R_{L}+R_{2}\right)^{2}-\frac{4 L}{C}\right]
$$

By combining Formulae (6) and (7), it is obtained that $4 L / C \leq\left(R_{L}+R_{2}\right)^{2} \leq 2 L / C$. As the two inequalities do not exist at the same time, \#2 in Formula (4) has no real solution. It means that when the transmit and receiving coils of the MCRWPT system have a large distance, there is one and only one resonant frequency, i.e. natural resonance frequency $\omega$, to make the Equation (4) valid. Under the condition, the system will not have frequency splitting. When there is a short transmission distance, that is, the mutual inductance between coils cannot be ignored relative to the self-inductance of the coils, to avoid the occurrence of frequency splitting in the MCRWPT system, the following condition needs to be satisfied:

$$
M<0.5\left(R_{L}+R_{2}\right) \sqrt{4 L C-C^{2}\left(R_{L}+R_{2}\right)^{2}}
$$

With given system parameters including load impedance, self-inductance of coils, matching capacitance, and internal resistance, the condition of not having frequency splitting changes to the condition that the mutual inductance between coils is less than a constant under certain parameter combination of the system. In other words, when coils in the system have a short distance, there is definitely a mutual inductance which causes the occurrence of frequency splitting. 
As the wireless power supply system of household appliances has a limited transmission distance, the mutual inductance needs to be effectively defined according to Formula (8) while designing the system, so as to avoid phenomena such as frequency splitting that difficult to deal with.

\section{ACCURATE CALCULATION OF MUtUAL INDUCTANCE AND COUPLING OPTIMIZATION}

The above analysis reveals that the mutual inductance between the coils is a significant factor determining whether frequency splitting occurs to the wireless power supply system of household appliances or not. Moreover, setting a proper mutual inductance is also an effect approach for guaranteeing the optimal transmission efficiency. To ensure that the theoretically designed mutual inductance matches with the actual inductance between coils, the mutual inductance between coils requires to be accurately calculated. An approximate calculation method for mutual inductance between spatial spiral coils was proposed in previous research [12], that is, the calculation formula for mutual inductance in multi-turn coil domains based on Neumann formula. The model of the coil is demonstrated in Figure II and the calculation expression is shown as Formula (9).

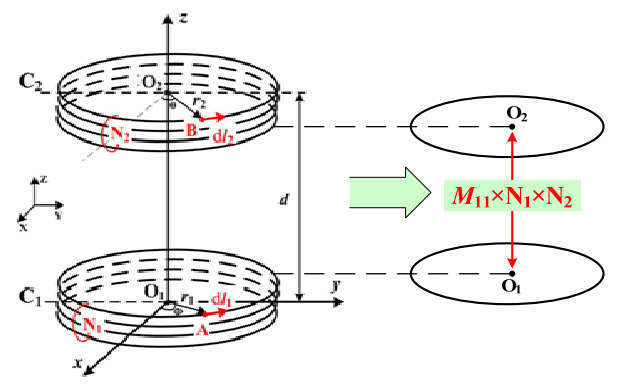

FIGURE II. CALCULATION MODEL FOR MUTUAL INDUCTANCE IN MULTI-TURN COIL DOMAINS BASED ON NEUMANN FORMULA

$$
\begin{aligned}
M & =N_{1} N_{2} \frac{\mu_{0}}{4 \pi} \oint_{C 1} \oint_{C 2} \frac{\mathrm{d} \ell_{1} \mathrm{~d} \ell_{2}}{|A-B|} \\
& =N_{1} N_{2} \frac{\mu_{0}}{4 \pi} \int_{0}^{2 \pi} \mathrm{d} \varphi \int_{0}^{2 \pi} \frac{r_{1} r_{2} \cos (\theta-\varphi) \mathrm{d} \varphi}{\sqrt{r_{1}^{2}+r_{2}^{2}-2 r_{1} r_{2} \cos (\varphi-\theta)+d^{2}}}
\end{aligned}
$$

where $\mathrm{C}_{1}$ and $\mathrm{C}_{2}$ represent the serial numbers of the two coils, $r_{1}$ and $r_{2}$ are the radii of the two coils, $\Phi$ and $\theta$ denote the angles between arbitrary points on $\mathrm{C}_{1}$ and $\mathrm{C}_{2}$ coils with $x$ axis, and $\mathrm{d}_{1}$ and $\mathrm{d} \boldsymbol{l}_{2}$ refer to the infinitesimal integral phasors of arbitrary points on the two coils. Moreover, $d$ denotes the distance between the two coils, and $N_{1}$ and $N_{2}$ are the numbers of turns of the two coils.

As shown in Figure II and Formula (9), the approximate method of multiplying single-turn inductance by the number of turns of each coil is generally used to calculate the mutual inductance between multi-turn spatial spiral coils, while the winding height of the coils are ignored. The method has little calculation amounts and is easy to realize. However, the calculated results have great errors with the actual values.
Therefore, while designing a wireless power supply system, the coils need to be calibrated through experiments according to specific performance indexes. So the method has less theoretical guiding significance.

Aiming at the above problem, a more accurate calculation method for mutual inductance between coils is proposed based on the Neumann formula. Taking spatial spiral coils and disc spiral coils as examples, the detailed sketch map is illustrated in Figure III.

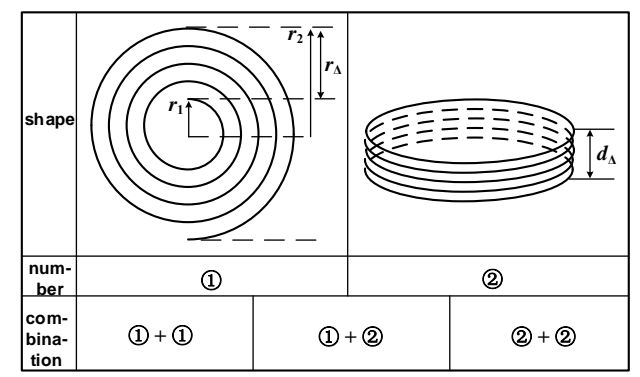

FIGURE III. CALCULATION MODEL FOR MUTUAL INDUCTANCE BETWEEN DIFFERENT COILS

There are two winding modes of wires in Figure III, where (1) and (2) represent the disc spiral coil and the spatial spiral coil, respectively, so there are three combination modes while designing the wireless power supply system for household appliances. In the figure, $r_{1}, r_{2}$, and $r_{\Delta}$ represent the inner radius, external radius, and their difference of the disc spiral coil, separately, and $d_{\Delta}$ denotes the height of the spatial spiral coil.

According to the Neumann formula, the coil radius and the transmission distance of wireless power supply systems are major variables determining the mutual inductance between coils. Taking spiral coils for an example, the actual mutual inductance between two spiral coils is less than the theoretical value calculated using Formula (9) due to the existence of the height of the coils. Therefore, the mutual inductance of power supply coils in different combination modes needs to be accurately calculated.

\section{A. Combination Mode (1)+(1)}

Suppose that the transmit and receiving coils of a wireless power supply system are $\mathrm{C}_{1}$ and $\mathrm{C}_{2}$ which have $N_{1}$ and $N_{2}$ turns and an initial radius of $r_{1}$. Moreover, the diameter of the winding wire is $a$, the space between turns is $q$, and the transmission distance is still $d$. Therefore, the mutual inductance between the coils can be represented as the cumulative sum of the mutual inductances of each turn of the coils, so

$$
\begin{aligned}
\sum_{j=1}^{N_{2}} M_{1 j} & =M_{11}+M_{12}+\cdots+M_{1 j}+\cdots+M_{1 N_{2}} \\
& =M\left(r_{1}, r_{1}\right)+M\left(r_{1}, r_{2}\right)+\cdots+M\left(r_{1}, r_{j}\right)+\cdots+M\left(r_{1}, r_{N_{2}}\right) \\
& =\sum_{j=1}^{N_{2}} M\left(r_{1}, r_{j}\right), \quad j \in\left[1, N_{2}\right]
\end{aligned}
$$


where $r_{j}=r_{1}+(j-1)(a+q)$. In this way, the mutual inductance between the two disc coils can be deduced as

$$
\begin{aligned}
M_{C_{1} C_{2}} & =\sum_{j=1}^{N_{2}} M\left(r_{1}, r_{j}\right)+\cdots+\sum_{j=1}^{N_{2}} M\left(r_{i}, r_{j}\right)+\cdots+\sum_{j=1}^{N_{2}} M\left(r_{N_{1}}, r_{j}\right) \\
& =\sum_{i=1}^{N_{1}} \sum_{j=1}^{N_{2}} M\left(r_{i}, r_{j}\right), \quad i \in\left[1, N_{1}\right]
\end{aligned}
$$

where $r_{i}=r_{1}+(i-1)(a+q)$.

\section{B. Combination Mode (1)+(2)}

Suppose that $\mathrm{C}_{1}$ and $\mathrm{C}_{2}$ represent a disc spiral coil and a spatial spiral coil which have $N_{1}$ and $N_{2}$ turns, respectively. The disc spiral coil has an initial radius of $r_{1}$ and a space of $q$ between turns. The initial radius and the space between turns of the spatial spiral coil are $\delta_{1}$ and $p$. In addition, the diameter of the winding wire is $a$ and the shortest distance between the two coils is $d$, so the mutual inductance between the two coils can be calculated using the following formula:

$$
\begin{aligned}
M_{C_{1} C_{2}} & =\sum_{j=1}^{N_{2}} M\left(r_{1}, d_{j}\right)+\cdots+\sum_{j=1}^{N_{2}} M\left(r_{i}, d_{j}\right)+\cdots+\sum_{j=1}^{N_{2}} M\left(r_{N_{1}}, d_{j}\right) \\
& =\sum_{i=1}^{N_{1}} \sum_{j=1}^{N_{2}} M\left(r_{i}, d_{j}\right), \quad i \in\left[1, N_{1}\right], j \in\left[1, N_{2}\right]
\end{aligned}
$$

where $r_{i}=r_{1}+(i-1)(a+q)$ and $d_{j}=d+(j-1)(a+p)$.

\section{Combination Mode (2)+(2)}

Suppose that the spatial spiral coils in this combination mode have the same parameters with that in the combination mode of (1)+(2). Therefore, the calculation formula for the mutual inductance is

$$
\begin{aligned}
M_{C_{1} C_{2}} & =\sum_{j=1}^{N_{2}} M\left(d_{1}, d_{j}\right)+\cdots+\sum_{j=1}^{N_{2}} M\left(d_{i}, d_{j}\right)+\cdots+\sum_{j=1}^{N_{2}} M\left(d_{N_{1}}, d_{j}\right) \\
& =\sum_{i=1}^{N_{1}} \sum_{j=1}^{N_{2}} M\left(d_{i}, d_{j}\right), \quad i \in\left[1, N_{1}\right], j \in\left[1, N_{2}\right]
\end{aligned}
$$

where $d_{i}=d+(i-1)(a+p)$ and $d_{j}=d+(j-1)(a+p)$.

\section{SIMULATION AND EXPERIMENTAL VERIFICATION}

To verify the correctness of the theoretical analysis and the accuracy of the calculation for the mutual inductance, a wireless power supply device for tail-free household appliances was designed. In addition, a LCR tester was used to measure the actual mutual inductance between coils in different combination modes. On this basis, the authors discussed the relation between the optimal transmission performance of the power supply device for tail-free household appliances based on mutual inductance coupling and the mutual inductance.
Besides, the correctness and accuracy of the proposed accurate calculation method were verified as well.

To verify the accuracy of the proposed expression for calculating the mutual inductance, the values calculated using Formulae (9) and (11) (13) were compared with the measured values. The experimental parameters are displayed in Table I.

TABLE I. PARAMETERS OF THE EXPERIMENTAL SYSTEM

\begin{tabular}{|c|c|c|c|c|}
\hline & $\begin{array}{c}\text { Number } \\
\text { of turns }\end{array}$ & $\begin{array}{c}\text { Inner } \\
\text { radius } \\
\text { (cm) }\end{array}$ & $\begin{array}{c}\text { Wire } \\
\text { diameter } \\
\text { (mm) }\end{array}$ & $\begin{array}{c}\text { Winding } \\
\text { pattern }\end{array}$ \\
\hline Coil mode (1) & $5 \sim 15$ & 15 & 4 & $\begin{array}{c}\text { Close } \\
\text { winding }\end{array}$ \\
\hline Coil mode (2) & $5 \sim 15$ & 20 & 4 & $\begin{array}{c}\text { Close } \\
\text { winding }\end{array}$ \\
\hline $\begin{array}{c}\text { Input voltage } \\
(\text { V) }\end{array}$ & \multicolumn{4}{|c|}{10} \\
\hline $\begin{array}{c}\text { Transmission } \\
\text { distance (cm) }\end{array}$ & \multicolumn{4}{|c|}{$24 \mathrm{~V} / 15 \mathrm{~W}$} \\
\hline Load (DVD) & \multicolumn{4}{|c|}{200} \\
\hline $\begin{array}{c}\text { Resonant } \\
\text { frequency (kHz) }\end{array}$ & \multicolumn{4}{|c|}{} \\
\hline
\end{tabular}

Under conditions with the above coil parameters, the mutual inductances between coils with different numbers of

\begin{tabular}{|c|c|c|c|c|c|c|}
\hline $\begin{array}{c}\text { Coil } \\
\text { mode }\end{array}$ & $\begin{array}{l}\text { Number } \\
\text { of turns }\end{array}$ & $\begin{array}{c}\text { Calculated } \\
\text { value } \\
\text { using } \\
\text { Formula } \\
\text { (9) }(\mu \mathrm{H})\end{array}$ & $\begin{array}{l}\text { Accurately } \\
\text { calculated } \\
\text { value }(\mu \mathrm{H})\end{array}$ & $\begin{array}{c}\text { Measured } \\
\text { value } \\
(\mu \mathrm{H})\end{array}$ & $\begin{array}{c}\text { Error } \\
1\end{array}$ & $\begin{array}{c}\text { Error } \\
2\end{array}$ \\
\hline \multirow{3}{*}{$\begin{array}{l}\text { (1) }+ \\
\text { (1) }\end{array}$} & 5 & 0.37 & 0.36 & 0.36 & $2.8 \%$ & $0 \%$ \\
\hline & 10 & 2.05 & 2.04 & 2.03 & $1 \%$ & $0.5 \%$ \\
\hline & 15 & 6.20 & 6.12 & 6.10 & $1.3 \%$ & $0.3 \%$ \\
\hline \multirow{3}{*}{$\begin{array}{l}\text { (1) }+ \\
\text { (2) }\end{array}$} & 5 & 0.48 & 0.43 & 0.40 & $20 \%$ & $7.5 \%$ \\
\hline & 10 & 2.28 & 1.85 & 1.83 & $24.6 \%$ & $1.1 \%$ \\
\hline & 15 & 5.94 & 4.36 & 4.30 & $38.1 \%$ & $1.4 \%$ \\
\hline \multirow{3}{*}{$\begin{array}{l}\text { (2) }+ \\
\text { (2) }\end{array}$} & 5 & 0.63 & 0.53 & 0.55 & $14.5 \%$ & $3.6 \%$ \\
\hline & 10 & 2.53 & 1.69 & 1.77 & $42.9 \%$ & $4.5 \%$ \\
\hline & 15 & 5.70 & 3.13 & 3.31 & $72.2 \%$ & $5.4 \%$ \\
\hline
\end{tabular}
turns were measured and compared with the theoretical value. In the experiment, the transmit and receiving coils always have same number of turns. The results are listed in Table II.

TABLE II. CALCULATED AND MEASURED MUTUAL INDUCTANCES IN DIFFERENT COIL MODES

In Table II, error 1 represents the absolute error between the value calculated using Formula (9) and the measured value, and error 2 is that between the accurately calculated value with the measured value. The comparison between the values calculated using different formulae and the measured value reveals that the traditional calculation formula has a large error with the measured value and therefore cannot accurately guide the parameter design of the wireless power supply system. This is 
because in the traditional calculation formula, the reference radius of coils and transmission distance are fixed when the coils have different numbers of turns. For this reason, the more the turns, the larger the error is. In comparison, all parameters of the proposed accurate calculation formula for mutual inductance are variables changing with the numbers of turns of coils, and therefore are more approximate to those of actual systems. Therefore, the calculated results using the proposed formula have a smaller error and can favorably guide the optimization design of the system, especially for the design of the mutual inductance in critical coupling involving the optimal transmission performance and the frequency splitting.

To more intuitively describe the changes of the mutual inductance with the numbers of turns of coils in wireless power supply systems, Figures VI(A), (B), and (C) show the changes of the mutual inductance in different combination modes of the coils.

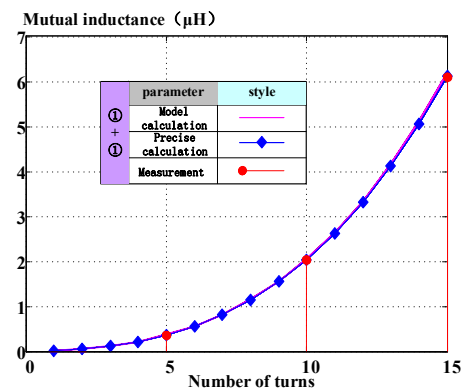

(A) COIL COMBINATION MODE (1)+(1)

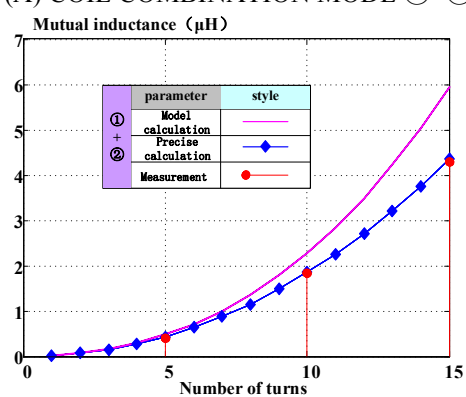

(B) COIL COMBINATION MODE (1)+(2)

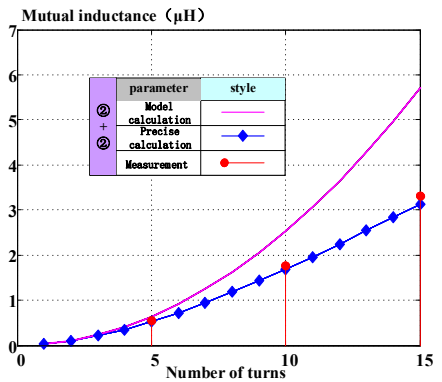

(C) COIL COMBINATION MODE (2)+(2)

FIGURE IV. RELATIONS BETWEEN MUTUAL INDUCTANCE AND
NUMBER OF TURNS IN DIFFERENT COMBINATION MODES OF COILS

By comparing the changes of mutual inductance in the three combination modes of coils in Figure IV, it is found that the mutual inductance between coils non-linearly augments with the increasing number of turns. In the combination mode of (1)
+1), the calculated values using the traditional formula are well consistent with the accurate results calculated using the proposed formula and the measured values. While in the other two combination modes, the calculated value using the traditional formula cannot guide the parameter design of the wireless power supply system, which well agrees with the results in Table II.

According to Formula (8), when the wireless power supply system has the optimal transmission efficiency, the mutual inductance needs to be smaller than a constant under certain parameter combination of the system. Under the condition, no frequency splitting occurs to the wireless power supply system. Owing to the traditional calculation formula has large errors while guiding the design of the actual system, which leads to inaccurate and deviated optimal transmission performance, it calls for multiple calibrations in the actual system design according to experience. In comparison, the proposed accurate calculation formula can preferably guide practical system design. Figures V to VII show the curves of transmission performances of the system in different combination modes of coils with parameters in Table I.

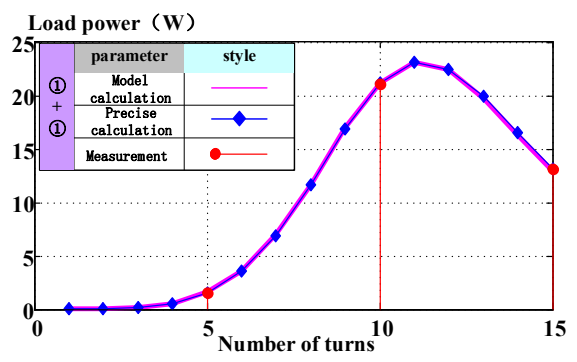

(A) CHANGE CURVE OF POWER WITH NUMBER OF TURNS OF COILS IN THE COMBINATION MODE OF (1)+(1) Transmitting efficiency

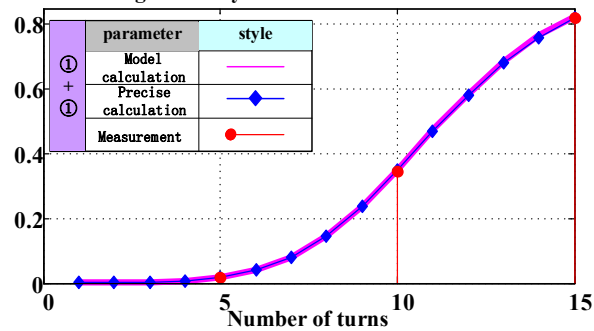

(B) CHANGE CURVE OF EFFICIENCY WITH NUMBER OF TURNS OF COILS IN THE COMBINATION MODE OF (1)+1)

FIGURE V. CHANGES OF POWER SUPPLY PERFORMANCE WITH TURNS OF COILS IN COMBINATION MODE OF (1)+(1)

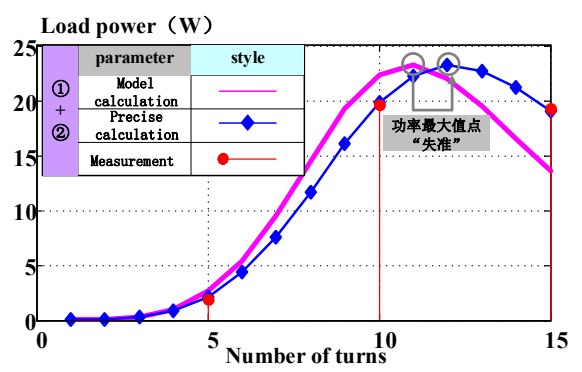

(A) CHANGE CURVE OF POWER WITH NUMBER OF TURNS OF COILS IN THE COMBINATION MODE OF (1)+(2) 
Transmitting efficiency

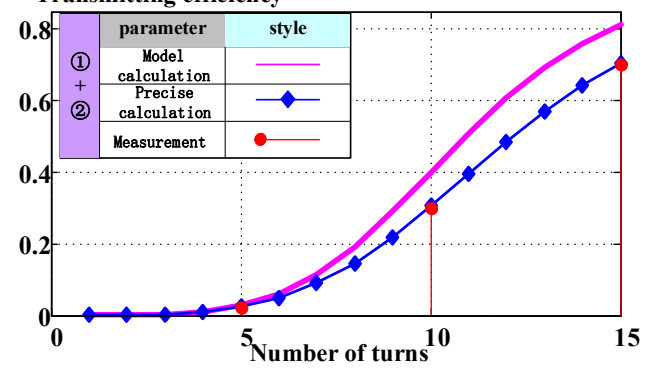

(B) CHANGE CURVE OF EFFICIENCY WITH NUMBER OF TURNS OF COILS IN THE COMBINATION MODE OF (1)+(2)

FIGURE VI. CHANGES OF POWER SUPPLY PERFORMANCE WITH TURNS OF COILS IN COMBINATION MODE OF (1)+(2)

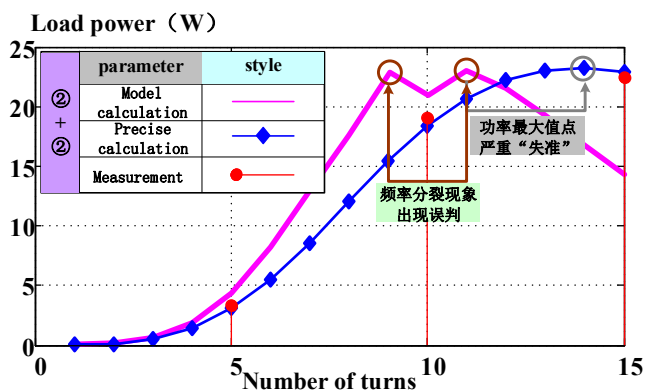

(A) CHANGE CURVE OF POWER WITH NUMBER OF TURNS OF COILS IN THE COMBINATION MODE OF (2)+(2) Transmitting efficiency

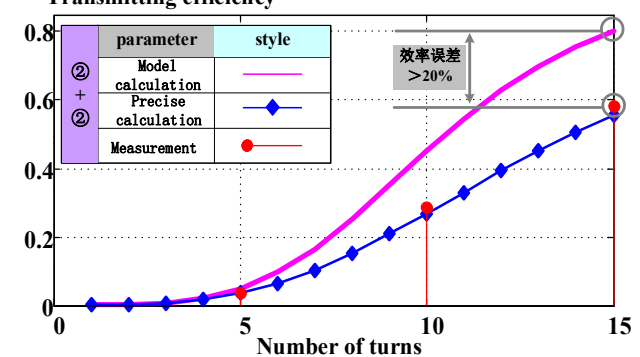

(B) CHANGE CURVE OF EFFICIENCY WITH NUMBER OF TURNS OF COILS IN THE COMBINATION MODE OF (2)+(2)

FIGURE VII. CHANGES OF POWER SUPPLY PERFORMANCE WITH TURNS OF COILS IN COMBINATION MODE OF (2)+(2)

It can be seen from the above change curves that the transmission efficiency of the system grows with the increasing number of turns of coils. When one or two spatial spiral coils (coil (2) ) exist in the wireless power supply system, the theoretical values obtained using the traditional calculation formula differ greatly from the experimental results and exert certain disturbance to the system design. Meanwhile, the mutual inductance and the power-supply power of loads do not absolutely monotonously augment, as shown in Figure V(A). In Figures VI(A) and VII(A), the points of maximum power are inaccurate and deviated, which is caused by the inaccurate calculation of the mutual inductance. Moreover, frequency splitting occurs to the power-supply power of loads calculated using the traditional formula in Figure VII(A). If the resonators of the power supply system of tail-free household appliances are designed according to the change curve, the power-supply power with planned number of turns ( 9 turns for instance) cannot meet the power demand for loads. Finally, experiments were carried out to verify the correctness of the theoretical analysis in the research and the experimental results were found to be basically consistent with the theoretical results obtained using the accurate calculation method proposed in the research.

\section{CONCLUSIONS}

Based on the significance of resonator design in elevating the performance of the power supply system of tail-free household appliances, the theoretical modeling method for circuits is adopted to analyze the coupling optimization of resonators. To get rid of the influences of the frequency splitting probably happening in the design process on the transmission performance of the power supply system, the analytic calculation is conducted to obtain the advisable domain value of mutual inductance that can avoid frequency splitting. On this basis, to obtain the accurate mutual inductance between resonators, the authors also carry out theoretical calculation in different combination modes of disc spiral coils and spatial spiral coils. The calculation method for mutual inductance more accurate than the traditional Neumann formula is proposed. Finally, the theoretical analysis in the research was verified through experimental measurement and comparison. The research is of great significance for improving the transmission performance of the power supply system of tail-free household appliances and avoiding the occurrence of frequency splitting.

\section{ACKNOWLEDGEMENT}

This research is supported by China Southern Power Grid Corporation (Research and Application of Key Technologies of Intelligent Cloud Service in Hainan Power Grid).

\section{REFERENCES}

[1] Huang Huiqun, Dong Xiulian, Li Dan, et al. Research on wireless transmission technology in the application of home appliance. China Appliance, 2012, 14(S1): 644-648(in Chinese).

[2] Huang Xueliang, Wang Wei, Tan Linlin, Technical progress and application development of magnetic coupling resonant wireless power transfer. Automation of Power Systems, 2017, 41(2): 2-14(in Chinese).

[3] TESLA N. The transmission of electric energy without wires. The Thirteenth Anniversary Number of the Electrical World and Engineer, Mar. 5, 1904, New York, USA.

[4] Kurs A, Karalis A, Moffatt R, et al. Wireless power transfer via strongly coupled magnetic resonances. Science, 2007, 317(5834): 83-86.

[5] Abdolkhani A, Hu A P, Moridnejad M, et al. Wireless charging pad based on travelling magnetic field for portable consumer electronics. 2013, 45(11): 1416-1421.

[6] Li Changsheng, Zhang He, Cao Juan, et al. Analysis and optimal design for power and efficiency transmission characteristics of magnetic resonance coupling power transmission systems. Automation of Electric Power Systems, 2015, 39(8): 92-97(in Chinese).

[7] Zhang Xian, Yang Qingxin, Cui Yulong, et al. Design optimization and verification on the power transmitting coil in the high-power wireless power transmission system. Transactions of China Electrotechnical Society, 2013, 28(10): 12-18(in Chinese).

[8] Nguyen V T, Kang S H, Choi J H, et al. Magnetic resonance wireless power transfer using three-coil system with single planar receiver for laptop applications. IEEE Transactions on Consumer Electronics, 2015, 61(2): 160-166. 
[9] Wang W, Huang X L, Guo J P, et al. Power stabilization based on efficiency optimization for WPT systems with single relay by frequency configuration and distribution design of receivers. IEEE Transactions on Power Electronics, 2017, 32(9): 7011-7024.

[10] Yeo T D, Kwon D S, Khang S T, et al. Design of maximum efficiency tracking control scheme for closed-loop wireless power charging system employing series resonant tank. IEEE Transactions on Power Electronics, 2017, 32(1): 471-478.

[11] Zhao Zhengming, Zhang Yiming, Chen Kainan. New progress of magnetically-coupled resonant wireless power transfer technology. Proceedings of the CSEE, 2013, 33(3): 1-13(in Chinese).

[12] Wang Wei, Huang Xueliang, Tan Linlin, et al. Effect analysis between resonator parameters and transmission performance of magnetic coupling resonant wireless power transmission system. Transactions of China Electrotechnical Society, 2015, 30(19): 1-6(in Chinese). 